\title{
Evidence for under-reported western European emissions of the potent greenhouse gas HFC-23
}

\author{
Christoph A. Keller, ${ }^{1}$ Dominik Brunner, ${ }^{1}$ Stephan Henne, ${ }^{1}$ Martin K. Vollmer, ${ }^{1}$ \\ Simon O’Doherty, ${ }^{2}$ and Stefan Reimann ${ }^{1}$ \\ Received 29 April 2011; revised 24 June 2011; accepted 27 June 2011; published 6 August 2011.
}

[1] Western European emission inventories of the potent greenhouse gas trifluoromethane (HFC-23) are validated at a country level by combining 2-hourly atmospheric in-situ measurements at Jungfraujoch (Switzerland) and Mace Head (Ireland) with Lagrangian transport simulations. HFC-23 has an atmospheric lifetime of $\sim 270 \mathrm{yr}$ and a $100-y r$ global warming potential (GWP) of 14,800 and is unintentionally produced during the manufacture of chlorodifluoromethane (HCFC-22). For the study region we derive emissions of $144-216 \mathrm{Mg} / \mathrm{yr}$ for July $2008-\mathrm{July}$ 2010 , which are $60-140 \%$ higher than the official emissions gathered from the national reports for the year 2009. The largest discrepancy is found for Italy, where our estimate of $26-56 \mathrm{Mg} / \mathrm{yr}$ exceeds the national inventory $(2.6 \mathrm{Mg} / \mathrm{yr})$ by more than an order of magnitude. These findings suggest that non-reported emissions from Annex 1 countries partly explain the recently derived gap between global bottom-up and top-down HFC-23 emission estimates. The results presented here provide independent information to relevant authorities on effective reporting of HFC-23 emissions, and demonstrate the potential of atmospheric measurements for real-world verification of greenhouse gas emissions. Citation: Keller, C. A., D. Brunner, S. Henne, M. K. Vollmer, S. O'Doherty, and S. Reimann (2011), Evidence for under-reported western European emissions of the potent greenhouse gas HFC-23, Geophys. Res. Lett., 38, L15808, doi:10.1029/ $2011 \mathrm{GL} 047976$.

\section{Introduction}

[2] Trifluoromethane (HFC-23, $\left.\mathrm{CHF}_{3}\right)$ is one of the most potent known greenhouse gases with an atmospheric lifetime of $\sim 270 \mathrm{yr}$ and a 100-yr global warming potential (GWP) of 14,800 [Forster et al., 2007]. Since its first reported measurements in 1978, its abundance has steadily increased in the atmosphere with Northern Hemispheric background concentration reaching 23 parts-per-trillion dry air mole fraction (ppt) in 2009 [Miller et al., 2010]. Apart from minor uses in air conditioners and fire extinguishers, HFC-23 emissions to the atmosphere primarily arise from its unavoidable production during the manufacture of chlorodifluoromethane $\left(\mathrm{HCFC}-22, \mathrm{CHClF}_{2}\right)$. The latter is widely

\footnotetext{
${ }^{1}$ Empa, Swiss Federal Laboratories for Materials Science and Technology, Duebendorf, Switzerland.

${ }^{2}$ Atmospheric Chemistry Research Group, School of Chemistry, University of Bristol, Bristol, UK.

Copyright 2011 by the American Geophysical Union. 0094-8276/11/2011GL047976
}

used as a cooling agent, for foam blowing, and as a feedstock for polymer production [McCulloch and Lindley, 2007; Miller et al., 2010].

[3] Due to its strong infrared-absorbing properties, HFC-23 was included in the Kyoto Protocol, which obligates the participating Annex 1 (developed) countries to reduce the emissions of a collection of greenhouse gases $\left(\mathrm{CO}_{2}, \mathrm{CH}_{4}\right.$, $\mathrm{N}_{2} \mathrm{O}$, HFCs, PFCs, and $\mathrm{SF}_{6}$ ) by $5.2 \%$ relative to 1990 emissions within 2008-2012. Compliance with the defined reduction targets is evaluated based on yearly emissions reported to the United Nations Framework Convention on Climate Change (UNFCCC). These emission estimates are mostly derived using bottom-up methods, which combine country-specific activity data with associated emission factors [Intergovernmental Panel on Climate Change, 2006]. The reliability of this approach is a matter of ongoing debate, and voices are rising that a thorough verification of reported emissions by atmospheric observations ('top-down') should be mandatory for a credible implementation of any greenhouse gas regulation [Levin et al., 2010; Nisbet and Weiss, 2010; Tollefson, 2010]. This is particularly true for the long-lived fluorinated compounds (F-gases: HFCs, PFCs and $\mathrm{SF}_{6}$ ) for which large bottom-up emission uncertainties of up to $100 \%$ have been stated (http://unfccc.int/national_reports/ annex_i_ghg_inventories/national_inventories_submissions/ items $/ \overline{5} 888$.php), mainly due to the poorly known emissions from banked substances. In these cases, real-world verification can largely help to ascertain compliance with the imposed emission reductions.

[4] Here, we estimate western European HFC-23 emissions at a country level by using two different top-down methods, linking atmospheric concentration observations at Jungfraujoch (Switzerland) and Mace Head (Ireland) with Lagrangian transport simulations. Subsequently, we compare these values to the emission numbers reported to the UNFCCC. The study is constrained to the region most strongly influencing the two measurement sites, and includes the United Kingdom (UK), Ireland, the Benelux countries (Belgium, the Netherlands, Luxembourg), Germany, Switzerland, France, Austria, and Italy.

[5] HFC-23 was purposely chosen for investigation. It has only few, but precisely localized emission sources which are, however, poorly quantified, and its atmospheric residence time greatly exceeds the time required to advect emissions towards the selected measurement sites. Both characteristics make HFC-23 very suitable for top-down emission quantification. Furthermore, unlike other long-lived HFCs for which there are plans to phase out their use and replace them by new synthetic compounds such as halogenated olefins, HFC-23 emissions are envisaged to continue along with the 


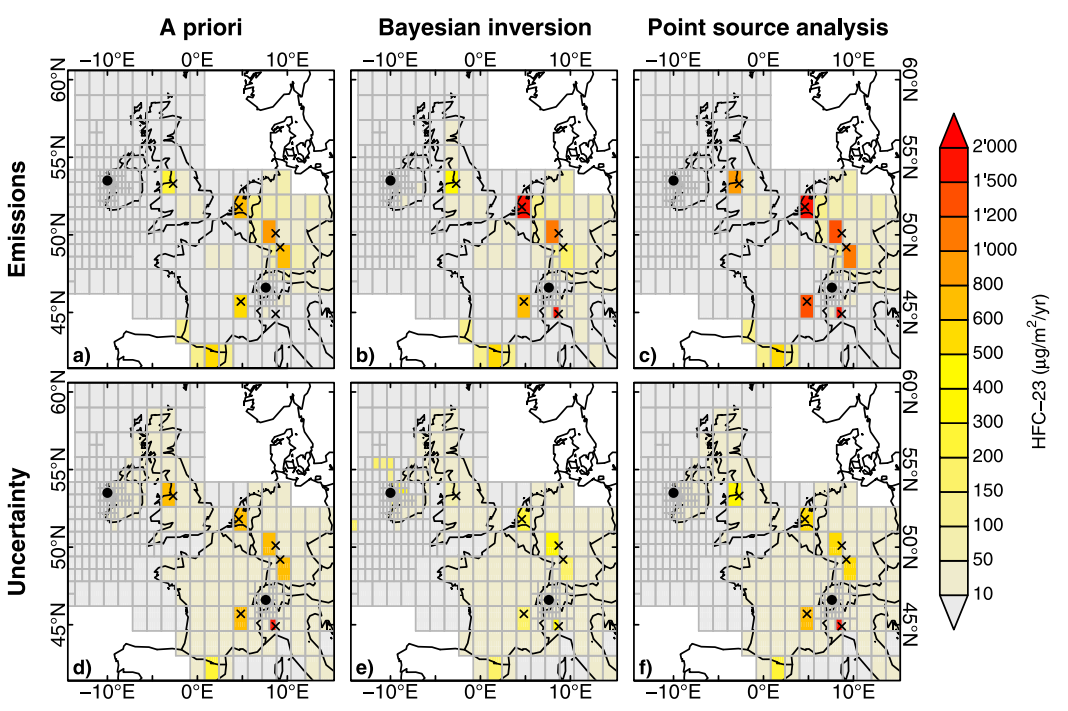

Figure 1. Maps of a priori and inversely determined HFC-23 emission rates $\left(\mu \mathrm{g} / \mathrm{m}^{2} / \mathrm{yr}\right)$ and corresponding uncertainties. (a) A priori emission map depicting the officially reported country emissions of 2009. (b and c) Bayesian inversion and (time variable) point source analysis emission maps, respectively, showing the optimized emission fluxes as determined by inverse modeling for the time period July 2008-July 2010. (d-f) Estimated uncertainties of a priori emissions, Bayesian inversion results and point source analysis results. Black dots show the measurement sites, and the crosses indicate locations of HCFC-22 production plants. For better visualization, HFC-23 emissions were omitted below $0 \mu \mathrm{g} / \mathrm{m}^{2} / \mathrm{yr}$ and above $2,000 \mu \mathrm{g} / \mathrm{m}^{2} / \mathrm{yr}$.

manufacture of polymer products, implying a continued climatic significance of HFC-23.

\section{Methods}

\subsection{Measurement Sites and Instruments}

[6] The monitoring sites Jungfraujoch (3,580 $\mathrm{m}$ above sea level) and Mace Head are located in the Central Swiss Alps and on the western coast of Ireland, respectively. Both stations are remote measurement sites episodically influenced by emissions in the European atmospheric boundary layer [Henne et al., 2010], and are embedded in the international measurement networks SOGE (System for Observation of Halogenated Greenhouse Gases in Europe), AGAGE (Advanced Global Atmospheric Gases Experiment), and WMO GAW (World Meteorological Organisation Global Atmosphere Watch). HFC-23 has been measured since November 2007 (Mace Head) and June 2008 (Jungfraujoch) every two hours by a gas chromatograph/mass spectrometer system coupled to a low-temperature pre-concentration unit ("Medusa") [Miller et al., 2008]. The measurements are reported on the SIO-07 primary calibration scale and measurement precision and the accuracy of the reported mole fractions are estimated at $0.6 \%$ and $\leq 3 \%$, respectively [Miller et al., 2010].

\subsection{Dispersion Modeling}

[7] The Lagrangian Particle Dispersion model FLEXPART v8.1 [Stohl et al., 2005] was used to obtain the source-receptor relationship (SRR), which is defined here as the residence times of the particles below $100 \mathrm{~m}$ above model ground, divided by the air density. It was driven by 3-hourly wind fields of the European Centre for Medium-range Weather Forecast wind fields (alternating analysis and 3 hour) of $1^{\circ} \times 1^{\circ}$ horizontal resolution (a nested grid of $0.2^{\circ} \times 0.2^{\circ}$ was used over the Alps) and 91 vertical levels. 50,000 particles were released every 3 hours between 0 to $10 \mathrm{~m}$ above model ground (Mace Head) and at 3,000 m above sea level (Jungfraujoch) and tracked 5 days backwards in time.

[8] To account for the reduced sensitivity with increasing distance from the measurement stations, the inversion grid size increases from $0.2^{\circ} \times 0.2^{\circ}$ close to the observational sites to $1.6^{\circ} \times 1.6^{\circ}$ at the limits of the covered domain. The ability of the FLEXPART calculations to reproduce surface residence times was tested by comparing the model predicted time series of a suite of reference compounds against the respective measurements, showing very good agreement between model and observations (Figure S1 of the auxiliary material). ${ }^{1}$

\subsection{Bayesian Inversion}

[9] The first inversion method, hereafter called 'Bayesian inversion', builds on least-square optimization and aims at minimizing both the differences between observed and model predicted concentrations and the deviation from an a priori emission field, assuming constant emissions in time [Stohl et al., 2009]. A priori emissions are compiled from the UNFCCC data (http://unfccc.int/national_reports/annex i_ghg_inventories/national_inventories_submissions/items/ 5888.php) and comprise HFC-23 emissions from HCFC-22 production assigned to the grid boxes containing the respective plants, and remaining emissions disaggregated by population density [CIESIN, 2005].

[10] The uncertainties attributed to the different inversion parameters were determined from emission field characteristics and statistical tests. Control runs show that the overall results are only weakly sensitive to the a priori definitions, but are primarily determined by atmospheric transport and the observations (Figures S2 and S3 of the auxiliary

${ }^{1}$ Auxiliary materials are available in the HTML. doi:10.1029/ 2011 GL047976. 
Table 1. Reported and Estimated Annual HFC-23 Emissions, in Mg, per Country ${ }^{\mathrm{a}}$

\begin{tabular}{lcccc}
\hline & & \multicolumn{2}{c}{ July 2008-July 2010 } \\
\cline { 3 - 5 } & 2009 Reported & A Priori & Bayesian Inversion & Point Source Analysis \\
\hline BLX & 13 & $13(0-29)$ & $36(31-41)$ & $41(26-46)$ \\
FRA & 15 & $15(0-30)$ & $27(22-34)$ & $33(31-58)$ \\
GER & $22-86^{\mathrm{b}}$ & $52^{\mathrm{c}}(31-73)$ & $40(32-48)$ & $63(51-84)$ \\
IT & 2.6 & $2.6(0-18)$ & $26(23-29)$ & $56(41-74)$ \\
UK & 5.5 & $5.5(0-21)$ & $12(10-14)$ & $21(13-29)$ \\
Countries without HCFC-22 production: & 1.6 & $1.6(0.6-2.6)$ & $2.0(1.2-2.8)$ & \\
AUT & 0.30 & $0.30(0-0.69)$ & $0.62(0.35-0.89)$ & \\
CH & 0.44 & $0.44(0-0.91)$ & $0.46(0-1.5)$ & \\
IRL & &
\end{tabular}

${ }^{a}$ Reported numbers for year 2009 are shown for Benelux (BLX), France (FRA), Germany (GER), Italy (IT), the United Kingdom (UK), western Austria (AUT), Switzerland (CH), and Ireland (IRL). Columns 3 and 4 give the Bayesian inverse modeling a priori and a posteriori emissions, respectively, as estimated for the time period July 2008-July 2010. Column 5 depicts the emission numbers for the same time period as derived from the point source analysis. Numbers in brackets indicate the uncertainty range.

${ }^{\mathrm{b}}$ The upper value includes maximal reported emissions from HCFC-22 production as extracted from total non-specified emissions.

${ }^{\mathrm{c}}$ Assuming emissions of $15 \mathrm{Mg}$ for each German HCFC-22 plant.

material). Compared to previous studies [Stohl et al., 2010; Vollmer et al., 2009], we extended the inversion algorithm according to Tarantola and Valette [1982] to provide a direct estimate of the posterior emission flux uncertainty (see auxiliary material).

\subsection{Point Source Analysis}

[11] The second inversion method accounts for the potentially large temporal variability of HFC- 23 emissions and takes advantage of the fact that HFC-23 emissions from HCFC-22 manufacture are well localized. We refer to this method as the '(time variable) point source analysis'. In this approach, the excess concentrations observed at the two measurement sites (defined as the concentration which cannot be explained by the a priori emission field) are assumed to originate uniquely from HCFC- 22 production, and are evenly assigned to those production plants with a modeled SRR above $500 \mathrm{sm}^{3} / \mathrm{kg}$, while the remaining grid boxes are left unchanged with respect to the $a$ priori field used in the Bayesian inversion. The implementation of this SRR threshold circumvents inappropriate emission calculations for cases where grid cell sensitivities are weak, and the SRR threshold value was chosen such that, for a typical HFC-23 emission rate of $3 \times 10^{-3} \mathrm{~g} / \mathrm{m}^{2} / \mathrm{yr}$, the given grid cell causes a detectable concentration increase of at least $0.2 \mathrm{ppt}$ at the receptor site.

[12] Production plant emissions are calculated for every observation individually (i.e. for every 3 hour interval), leading to a comprehensive set of time-dependent emission estimates for every HCFC-22 production site. These data follow a log-normal distribution (Figure S4 of the auxiliary material), and we therefore assigned the fitted value of the log-normal distribution to those periods where residence times are below the SRR threshold. We performed several sensitivity tests to check the robustness of the results against the definition of the grid box sizes and the choice of the SRR threshold, and used these results to estimate a conservative uncertainty range of the derived emissions (Figure S5 of the auxiliary material).

\section{Discussion}

[13] The emission distributions as inferred from the two employed methods are depicted in Figure 1 and detailed in
Table 1. It is important to emphasize that the Bayesian inversion likely underestimates real HFC-23 emissions as it gives low weight to those observations with a large mismatch between measured and modeled a priori concentration, such that the modeled posterior time series are not able to adequately reproduce the observed large HFC-23 perturbations (see Figure 2). The point source analysis, on the other hand, is likely to place an upper bound on real emissions as this method is more susceptible to inaccuracies in individual transport model calculations and tends to overestimate the emissions in cases where the surface residence times are underestimated by the model. The two methods used are therefore expected to place a lower and upper constraint on HFC-23 emissions rather than providing mean flux estimates.

[14] Total annual HFC-23 emissions derived from the Bayesian inversion and the time variable point source analysis amount to $144(120-171)$ and $216(163-295) \mathrm{Mg} / \mathrm{yr}$,

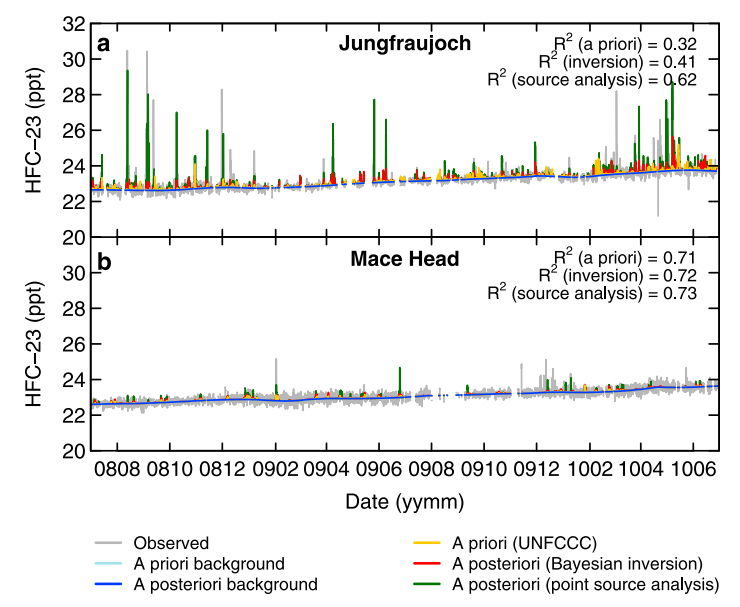

Figure 2. HFC-23 time series of observations (grey), background signal (blue), and model-predicted prior (yellow) and posterior concentrations determined from the Bayesian inversion (red) and the point source analysis (green), respectively, at (a) Jungfraujoch and (b) Mace Head between July 2008 and June 2010. Correlations with observations are given on top right. 

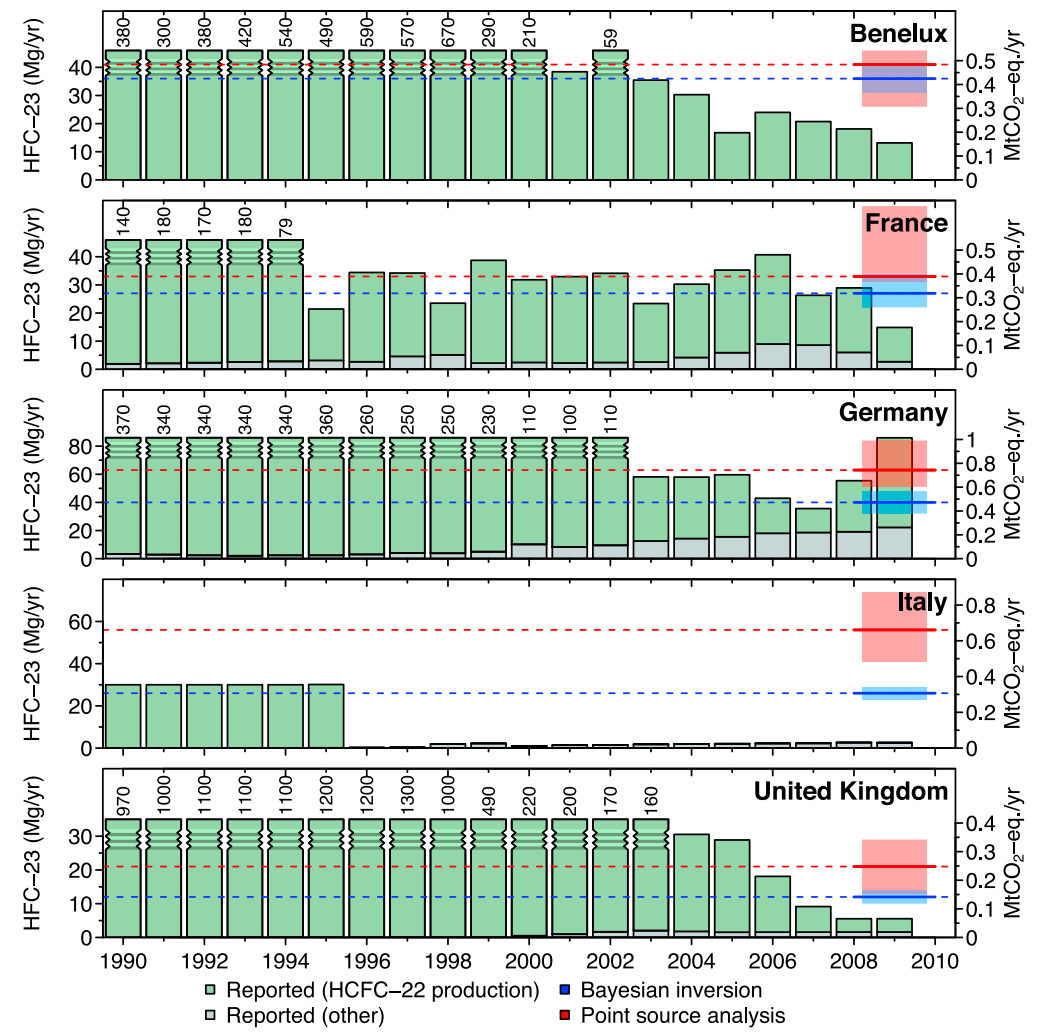

Figure 3. Comparison of reported and estimated HFC- 23 emissions of principal emitting countries in western Europe. Annual emissions reported to UNFCCC since 1990 are shown in green (emissions from HCFC-22 production) and grey (other emissions). Note the different y-axis and that bars are shrunk for high reported values, with total reported emissions indicated by the numbers above the respective bars. German emissions from HCFC-22 production were derived from values reported as total non-specified and thus provide an upper estimate of the reported value. Inversely estimated annual emissions between July 2008 and July 2010 are given by the solid blue line, with the corresponding uncertainties shaded in light blue. Solid red lines and reddish area indicate estimated average emissions and associated uncertainties as inferred from the point source analysis.

respectively, with similar contributions from each HCFC-22 production plant (Table 1 and Figure 1). The comparatively low emissions of $12-21 \mathrm{Mg} / \mathrm{yr}$ estimated from the UK plant agree with the fact that this site has only been in operation during parts of the study period [MacCarthy et al., 2011]. This is also deducible from the small fluctuations in the Mace Head observations, which indicate only small HFC-23 sources within the region of influence of this site (Figure 2).

[15] For most countries, our estimates are considerably larger than the emissions reported within the framework of the Kyoto Protocol, questioning the credibility of these HFC-23 emission inventories. For instance, our results indicate a strong contribution of $26-56 \mathrm{Mg} / \mathrm{yr}$ from northern Italy, suggesting that this region is one of the major HFC-23 sources within the study area (Figure 1). This is contrary to the Italian inventory which quotes only marginal HFC-23 emissions of $2.6 \mathrm{Mg} / \mathrm{yr}$. Given the high GWP of HFC-23, the unreported Italian emissions derived in this study translate to 274-625 kt $\mathrm{CO}_{2}$-equivalents (taking a GWP of 11,700 as approved in the Kyoto Protocol), implying that these missing HFC-23 emissions alone account for 34\%$77 \%$ of the indicated overall uncertainty of Italian F-gas emissions of $814 \mathrm{kt} \mathrm{CO}_{2}$-eq [Romano et al., 2011]. Since similar inconsistencies were also deduced for other compounds [Greally et al., 2007; Reimann et al., 2008], it appears likely that the official Italian F-gas inventory significantly underestimates the real emissions.

[16] The Italian inventory furthermore indicates that since 1996, HFC-23 emissions have been well below the 26$56 \mathrm{Mg} / \mathrm{yr}$ estimated by our study (Figure 3). By assuming that real emissions have never fallen below the 2008-2010 level (indicated by the dashed red and blue line in Figure 3), this would imply that non-reported HFC- 23 emissions from Italy have amounted to 3,700-10,700 kt $\mathrm{CO}_{2}$-eq. since 1996.

[17] Although to a smaller degree, similar considerations also apply for the Benelux countries and the UK, where our estimates indicate an amount of up to 1,500 and $530 \mathrm{kt} \mathrm{CO}_{2}$-eq. presumably not reported since 2003 and 2007, respectively. For France, our results are consistent with the reported emissions between 1995 and 2008 and hence do not support the reported strong emission decrease between 2008 and 2009. The interpretation is more complicated for Germany, where HFC-23 emissions from HCFC-22 production are not explicitly specified but given in conjunction with other confidential data. This results in a wide possible range of reported HFC-23 emissions of $22-86 \mathrm{Mg} / \mathrm{yr}$ in 2009 , which compares well with our estimates of $40-63 \mathrm{Mg} / \mathrm{yr}$ (Table 1). However, it is worth emphasizing that reported non-specified emissions are notably lower for 2003-2008, which could point to an underestimation of HFC-23 emissions by as much as $800 \mathrm{Mg}$ 
unless emissions from HCFC-22 production have more than doubled within this time period (Figure 3 ).

\section{Conclusions}

[18] HFC-23 emissions, as inferred from high-quality atmospheric measurements of this species, are up to an order of magnitude higher for individual western European countries than reported within the framework of the Kyoto Protocol, offering evidence for an under-reporting of HFC23 emissions in western Europe by $60-140 \%$. These excess emissions can mainly be attributed to releases from HCFC22 production in Italy and the Netherlands, reflecting the susceptibility of the HFC-23 budget to non-reported leakages in individual production plants.

[19] The here derived 54-126 Mg/yr of non-reported western European emissions represent 3-7\% of the recently reported gap between global top-down and bottom-up HFC-23 emission estimates of $1,900 \mathrm{Mg} / \mathrm{yr}$ for 2008 [Miller et al., 2010]. Given that the study area only covers $\sim 3 \%$ of total reported Annex 1 HFC-23 emissions and assuming that similar inconsistencies also exist in these inventories, it may be hypothesized that non reported emissions from the other Annex 1 countries explain a notable fraction of the remaining difference. This points towards the general problem that greenhouse gas emissions could potentially be overestimated for non-Annex 1 countries when these numbers are assessed on the basis of global top-down emission estimates corrected for Annex 1 emissions taken from the national inventories [e.g. Levin et al., 2010; Montzka et al., 2009; Mühle et al., 2010].

[20] Altogether, our work demonstrates the importance of an independent validation of reported emissions data, and it corroborates the potential of top-down methods to assess greenhouse gas emissions with high spatial and temporal resolution and a sufficient accuracy of 30-50\%. In particular as long as non-Annex 1 countries are not obliged to report their emissions to the UNFCCC, these methods provide the only tool to independently verify the Kyoto reporting process, demonstrating that future control mechanisms of any climate mitigation strategy should strive for a combination of bottom-up and top-down methods.

[21] To better constrain the global distribution of greenhouse gas emissions by means of atmospheric observations, a denser network of high quality measurements is needed, particularly in regions that are poorly covered at present, such as Eastern Europe and the East Asian region.

[22] Acknowledgments. We thank Winfried Schwarz, Öko-Recherche, for providing valuable information on the location of HCFC-22 plants. The measurements at Jungfraujoch are part of the Swiss National Air Pollution Monitoring Network (NABEL) and the HALCLIM-project and are supported by the Swiss Federal Office for the Environment (FOEN). This project was funded by the Swiss National Science Foundation (project 200021117753, 200020-125092/1, and 200021-120238). We also thank the Physics Department, National University of Ireland, Galway, for making the research facilities at Mace Head available. The operation of the Mace Head station was supported by the Department of the Energy and Climate Change (DECC, UK) (contracts GA01081 and GA01103 to the University of Bristol).

[23] The Editor thanks two anonymous reviewers for their assistance in evaluating this paper.

\section{References}

CIESIN (2005), Gridded population of the world: Future estimates, Socioeconomic Data and Applications Center (SEDAC), http://sedac.ciesin. columbia.edu/gpw, Columbia Univ., Palisades, New York.

Forster, P., et al. (2007), Changes in atmospheric constituents and in radiative forcing, in Climate Change 2007: The Physical Science Basis. Contribution of Working Group I to the Fourth Assessment Report of the Intergovernmental Panel on Climate Change, edited by S. Solomon et al., pp. 129-234, Cambridge Univ. Press, New York.

Greally, B. R., et al. (2007), Observations of 1,1-difluoroethane (HFC-152a) at AGAGE and SOGE monitoring stations in 1994-2004 and derived global and regional emission estimates, J. Geophys. Res., 112, D06308, doi:10.1029/2006JD007527.

Henne, S., et al. (2010), Assessment of parameters describing representativeness of air quality in-situ measurement sites, Atmos. Chem. Phys., 10, 3561-3581, doi:10.5194/acp-10-3561-2010.

Intergovernmental Panel on Climate Change (2006), General Guidance and Reporting, vol. 1, 2006 IPCC Guidelines for National Greenhouse Gas Inventories, edited by H. S. Eggleston et al., Inst. for Global Environ. Strategies, Tokyo, Japan.

Levin, I., et al. (2010), The global SF6 source inferred from long-term high precision atmospheric measurements and its comparison with emission inventories, Atmos. Chem. Phys., 10, 2655-2662, doi:10.5194/acp-102655-2010.

MacCarthy, J., et al. (2011), UK Greenhouse Gas Inventory, 1990 to 2009, AEA Technol. Plc., Oxfordshire, UK.

McCulloch, A., and A. A. Lindley (2007), Global emissions of HFC-23 estimated to year 2015, Atmos. Environ., 41, 1560-1566, doi:10.1016/ j.atmosenv.2006.02.021.

Miller, B. R., et al. (2008), Medusa: A sample preconcentration and GC/ MS detector system for in situ measurements of atmospheric trace halocarbons, hydrocarbons, and sulfur compounds, Anal. Chem., 80(5), 1536-1545, doi:10.1021/ac702084k.

Miller, B. R., et al. (2010), HFC-23 (CHF3) emission trend response to HCFC-22 (CHClF2) production and recent HFC-23 emission abatement measures, Atmos. Chem. Phys., 10, 7875-7890, doi:10.5194/acp-107875-2010.

Montzka, S. A., et al. (2009), Recent increases in global HFC-23 emissions, Geophys. Res. Lett., 37, L02808, doi:10.1029/2009GL041195.

Mühle, J., et al. (2010), Perfluorocarbons in the global atmosphere: Tetrafluoromethane, hexafluoroethane, and octafluoropropane, Atmos. Chem. Phys., 10, 5145-5164, doi:10.5194/acp-10-5145-2010.

Nisbet, E., and R. Weiss (2010), Top-down versus bottom-up, Science, 328(5983), 1241-1243, doi:10.1126/science.1189936.

Reimann, S., et al. (2008), Observations of long-lived anthropogenic halocarbons at the high-Alpine site of Jungfraujoch (Switzerland) for assessment of trends and European sources, Sci. Total Environ., 391(2-3), 224-231, doi:10.1016/j.scitotenv.2007.10.022.

Romano, D., et al. (2011), Italian Greenhouse Gas Inventory 1990-2009, Inst. for Environ. Protect. and Res., Rome, Italy.

Stohl, A., et al. (2005), Technical note: The Lagrangian particle dispersion model FLEXPART version 6.2, Atmos. Chem. Phys., 5, 2461-2474, doi:10.5194/acp-5-2461-2005.

Stohl, A., et al. (2009), An analytical inversion method for determining regional and global emissions of greenhouse gases: Sensitivity studies and application to halocarbons, Atmos. Chem. Phys., 9, 1597-1620, doi:10.5194/acp-9-1597-2009.

Stohl, A., et al. (2010), Hydrochlorofluorocarbon and hydrofluorocarbon emissions in East Asia determined by inverse modeling, Atmos. Chem. Phys., 10, 3545-3560, doi:10.5194/acp-10-3545-2010.

Tarantola, A., and B. Valette (1982), Generalized nonlinear inverse problems solved using least square criterion, Rev. Geophys., 20, 219-232, doi:10.1029/RG020i002p00219.

Tollefson, J. (2010), Greenhouse-gas numbers up in the air, Nature, 465(7294), 18-19, doi:10.1038/465018a.

Vollmer, M. K., et al. (2009), Emissions of ozone-depleting halocarbons from China, Geophys. Res. Lett., 36, L15823, doi:10.1029/2009GL038659.

D. Brunner, S. Henne, C. A. Keller, S. Reimann, and M. K. Vollmer, Empa, Swiss Federal Laboratories for Materials Science and Technology, Ueberlandstr. 129, Duebendorf CH-8600, Switzerland. (christoph.keller@ empa.ch)

S. O'Doherty, Atmospheric Chemistry Research Group, School of Chemistry, University of Bristol, Bristol BS8 1TS, UK. 